\title{
Echocardiography and cardiac biomarkers in patients with lung cancer treated with platinum-based chemotherapy
}

\author{
Daniel Omersa*, Tanja Cufer, Robert Marcun, Mitja Lainscak \\ University Clinic of Respiratory and Allergic Diseases Golnik, Golnik, Slovenia
}

Objective: Platinum-based chemotherapy is a standard regiment for advanced lung cancer and adjuvant therapy in an early stage. It is ototoxic, nephrotoxic and induces oxidative stress whilst the data regarding cardiotoxicity remain scarce. Our aim was to assess cardiotoxicity of the platinum-based chemotherapy in patients with lung cancer.

Patients and Methods: Patients with lung cancer referred for the first-line platinum-based chemotherapy were included in the study. Ultra-sensitive troponin T (usTnT), N-terminal pro-BNP (NT-proBNP) and echocardiography were performed at baseline, at the end of chemotherapy (visit two) and at the follow-up (visit three). We report mean \pm standard deviation and the number (percentage) for numeric and categorical variables, respectively. Elevated usTnT and NTproBNP was defined as $\geq 30 \%$ increase from the baseline. Significant left ventricular ejection fraction (LVEF) was defined as a decrease of LVEF $\geq 10 \%$ to value $\leq 55 \%$ whereas diastolic dysfunction was defined using the European Society of Cardiology guidelines.

Received: $20^{\text {th }}$ Apr 2014

University Clinic of Respiratory and Allergic Diseases Golnik, 4204, Golnik, Slovenia.

Phone: +386-4-2569-554

E-mail: daniel.omersa@klinika-golnik.si
Results: Overall, 41 patients (61 \pm 9 years, $54 \%$ men) were included. Patients received $5 \pm 1$ cycles of chemotherapy while $13(32 \%)$ had early stage lung cancer and received adjuvant chemotherapy. During the follow-up, 1 patient died before visit two, whereas 8 patients were not assessed at visit three ( 2 died, 6 were lost to follow-up). At baseline none of patients had overt heart failure, 4 had ischemic heart disease and one had peripheral obstructive arterial disease present. Values at baseline, visit two and visit three for usTnT, NT-proBNP and LVEF were $0.011 \pm 0.005 \mathrm{pg} / \mathrm{ml}$, $0.011 \pm 0.005 \mathrm{pg} / \mathrm{ml}, 0.008 \pm 0.003 \mathrm{pg} / \mathrm{ml}, 266.4 \pm 250.1 \mathrm{pg} / \mathrm{ml}$, $257.7 \pm 378.1 \mathrm{pg} / \mathrm{ml}, 225.9 \pm 430.0 \mathrm{pg} / \mathrm{ml}$ and $68 \% \pm 8 \%, 67 \% \pm$ $8 \%, 68 \% \pm 9 \%$, respectively. Diastolic dysfunction was found in $9(27 \%), 6(27 \%)$ and $4(24 \%)$ patients at baseline, visit two and visit three, respectively. From the baseline, significant elevation of usTnT and NT-proBNP to visit two and three was found in $3(16 \%), 2(25 \%)$ and 6 (35\%), 2 (25\%) patients, respectively, but none of the patients developed overt heart failure. A significant reduction of LVEF from baseline was observed in one patient at visit three.

Conclusions: In patients with lung cancer, platinum-based chemotherapy did not induce clinically relevant cardiovascular disease. In some patients, usTnT and NT-proBNP changes suggest subclinical cardiac injury, thus further research is warranted.

KEYWORDS: cardiotoxicity, chemotherapy, lung cancer, cisplatin, cardiac markers.

CITATION: Cardiol Croat. 2014;9(5-6):241. 